\title{
Modeling the Processes of Packet Losses in Telecommunication Networks in Program Anylogic
}

\author{
Azamat Qodirov \\ Data Communication Networks and Systems, Telecommunication Faculty, Tashkent University of Information Technologies, Tashkent, \\ Uzbekistan
}

Email address:

Tuit2013@umail.uz

To cite this article:

Azamat Qodirov. Modeling the Processes of Packet Losses in Telecommunication Networks in Program Anylogic. Engineering Physics. Vol. 2, No. 1, 2018, pp. 1-5. doi: 10.11648/j.ep.20180201.11

Received: March 6, 2018; Accepted: March 27, 2018; Published: April 20, 2018

\begin{abstract}
This paper deals with assessing and comparisons the real it is known for everyone that nowadays the services of the telecommunication networks are being developed day-to-day. The acceptable way of transmission data with the high quality, effect and safety through the network is to use the packet switching in the network. The main part of telecommunication networks is considered communication links. Through these communication links the information in the shape of packets is transmitted and during the transmission these packets pass some intermediate nodes. These intermediate nodes contain routers or switches, etc. By those intermediate nodes a lot of packet streams coming to the different nodes pass and in order not to be served to them at the same time the packets are curried in buffer in a queue. Then they are served in turn. In this condition packet loss and delay processes occur at intermediate nodes and this induces the decrease of the quality of service (QoS) in the network. This paper deals with the processes of packet losses occured in telecommunication networks in detail. Moreover, while researching the processes of packet losses, this network is modeled in AnyLogic program.
\end{abstract}

Keywords: Packet Loss, Circuit Switching, Buffer, Router, Packet Delay, Variance

\section{Introduction}

Communication networks are the support of the transmission of various types of information: voice, computer files, etc. They are constituted of nodes (machines, call servers, etc.) and links transporting the information between the nodes. Two main categories of communication networks can be distinguished. In circuit switching networks, used historically for telephony or currently in optical networks, an isolated channel is reserved between two nodes for the duration of the call. This way, once the connection is established, the transmission quality is guaranteed during the entire call. However, if no channel is available, a connection request might be blocked. The procedure which decides whether a request can be accepted or not is called admission control. On the other hand, in packet switching networks, consistent pieces of information (files for instance) are fragmented in packets emitted successively, that can take different routes to their destination and be multiplexed with packets from other sources. In such networks, even if a logical connection (e.g. a TCP connection) can be established between a source and a destination, there is no guarantee a priori on the links' availability, and subsequent packets might be lost or delayed. To limit packet losses, intermediate points of the network (routers and switches) can store a limited amount of packets in buffers. The well-known Internet is based on this packet-switching principle. It has greatly expanded over the last decade, and is still expanding fast.

Packet loss is the discarding of packets in a network when a router or other network device is overloaded and cannot accept additional packets at a given moment.

Packets are the fundamental unit of information transport in all modern computer networks, and increasingly in other communications networks as well.

The losses are usually due to congestion on the network and buffer overflows on the end-systems A buffer is a portion of a computer's memory that is set aside as a temporary holding place for data that is being sent to or received from an external device. A buffer overflow occurs any time more information is written into the buffer than there is space allocated for it in the memory.

Packet loss may or may not be disruptive to the recipient of the data, depending on the type of network service and the 
severity of the loss. With best effort services, packet loss is acceptable because recovery of the lost packets is handled by other services.

In networks, packets that have to be routed from one node to the other may have to be relayed through a series of intermediate nodes. Also, each node in the network may receive packets via many data streams that are being routed simultaneously from their source nodes to their respective destinations. In such conditions, the packets may have to be stored at intermediate nodes for transmission at a later time. If buffers are unlimited, the intermediate nodes need not have to reject or drop packets that arrive. For practical reasons, buffers are limited in size. Although a large buffer size is preferred to minimize packet drops, large buffers have an adverse effect on the mean and variance in packet delay.

\section{Method}

In wired communication networks, packet loss is almost solely caused by buffer overflow intermediate network nodes. Therefore, the packet loss ratio - the fraction of packets that get lost - is well studied in queueing literatures. However, in the case of multimedia communications, the packet loss ratio is not the only loss characteristic of interest. Multimedia applications can typically tolerate a reasonable amount of packet loss, as long as there are no bursts of packet loss. For example, the perceived visual quality of variable bit rate video streaming heavily depends on the burstiness of the packet loss process. Forward error correction (FEC) techniques may further mitigate the effects of packet loss and increase the tolerable packet loss ratio but again require that packet loss is well spread in time.

In telecommunication networks packet loss processes mainly occurs in two conditions (Figure 1). They are as follow:

1. queue (aka buffer) preceding link in buffer has finite capacity

2. packet arriving to full queue dropped (aka lost)

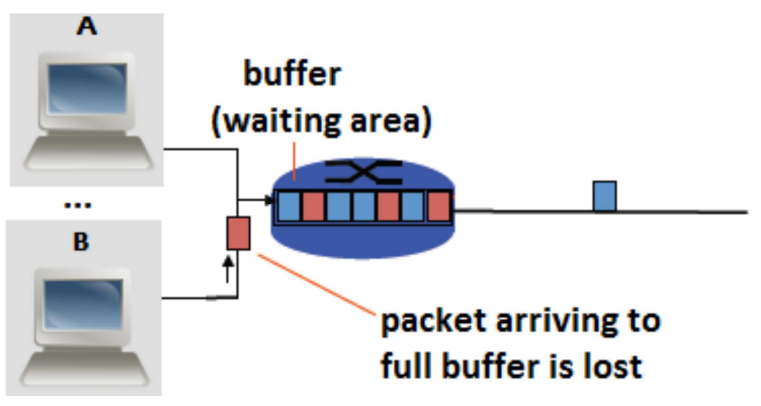

Figure 1. Two conditions of packet loss.

The sources of loss in telecommunication networks can be associated with the main elements of the network infrastructure: the routers and the links interconnecting the routers.

\subsection{Sources of Loss in the Routers}

If the load is higher than the forwarding speed of the routers, the packets are temporarily stored in the buffers of the routers, waitingfor their turn to beforwarded on the output link. A typical router can be represented as in Figure 2 [12]. It consists of input and output ports, a switch fabric and a routing processor. The packets waiting to be switched at the input ports are stored in the input port buffers while switched packets waiting to exit on the output link are stored in the output port buffers. The size of these buffers is finite. If the buffers are full, any new incoming packet is dropped. In other words, packets are lost due to buffer overflow.

Buffer overflow is the main cause of loss in wireline networks accounting for more than $99 \%$ of all the lost packets [13]. It is noted that packet loss due to buffer overflow is a consequence of limited buffer size and congestion in the network. The congestion in the network, and thus the necessary condition for having packet loss, is not resolved by only increasing the buffer memory $[14,15]$. Nagle shows that even with an infinite buffer size, packet loss is not eliminated [15]. Routers with infinite buffers (assuming that such routers exist) would drop packets not because of lack of space in the buffer, but because of expiration of the time-to-live (TTL) in the header of the IP packets.

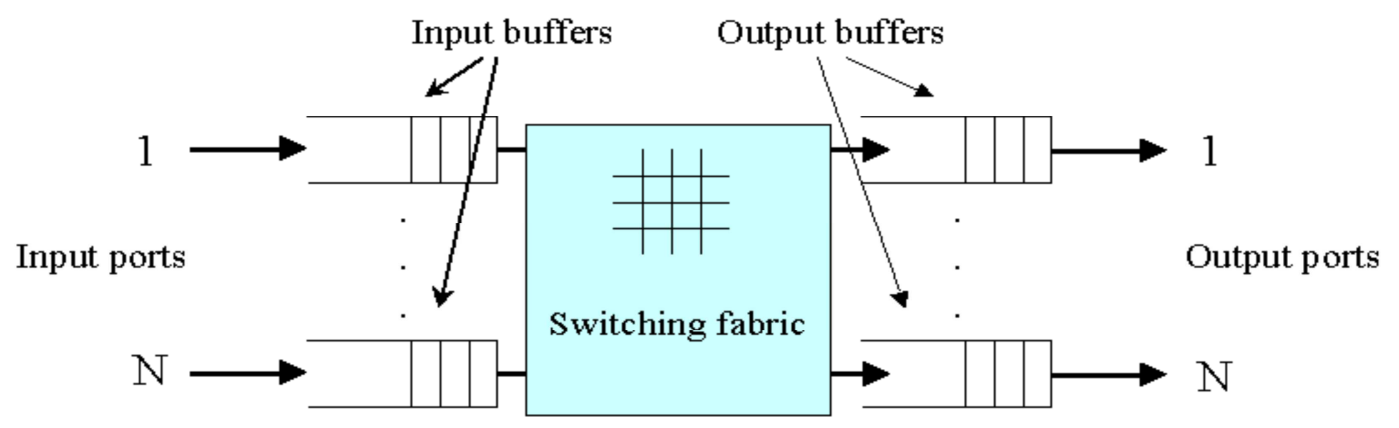

Processor

Figure 2. Generic schematic of a router.

Routing pathologies such as route loops [16] may also drops. cause expiration of the TTL field and consequently packet 


\subsection{Sources of Loss on the Links}

Link failures and lossy links are another cause of packet loss. For example, a typical wireless link often has high biterror rates due to channel fading, noise or interference, and intermittent connectivity due to handoffs. Corrupted packets might be dropped at any of the hops on the end-to-end path or at the receiver. Although sophisticated coding and checksum schemes exist for detection and correction of biterrors, unrecoverable packet corruption might occur, which is equivalent to a packet drop $[4,16,17]$.

The services in telecommunication networks are being developed day-to-day. The main part of telecommunication networks are considered communication links. Through these communication links the information in the shape of packets is transmitted and during the transmission these packets pass some intermediate nodes. These intermediate nodes contain routers or switches, etc. By those intermediate nodes a lot of packet streams coming to the different nodes pass and in order not to be served to them at the same time the packets are curried in buffer in a queue. Then they are served in turn. In this condition packet loss processes occur at intermediate nodes. In addition, packet loss may induce the general impression of a time delay, and thus, bias the perceived onset of the visual event. Higher packet loss rates give rise to the perception of longer time delays. Furthermore, the processes of packet loss cause to deteriorate quality of service of telecommunication networks. In buffers in the intermediate nodes packets could be loss in two ways: the first way is due to the buffer overflow; the second one is due to the delays in buffers. It is needed to create the mathematical and imitation models of telecommunication networks so as to evaluate accurately the characteristics of the existing networks. it gives us to see and predict the network faults (i.e. packet loss, packet delay variance, etc.) and helps to avoid before they occur.

In this paper the simulated model of the networks which has finite $(\mathrm{M} / \mathrm{M} / \mathrm{1} / \mathrm{r})$ buffers is shown which is simulated in program AnyLogic and obtained results related to the packet losses of them are compared by using the line graph (Figure $5)$.

Internet routers are packet switches, and therefore buffer packets during times of congestion. Arguably, router buffers are the single biggest contributor to uncertainty in the Internet. Buffers cause queueing delay and delay-variance; when they overflow they cause packet loss, and when they underflow they can degrade throughput.

\section{Result}

Table 1. The classes of packet loss characteristics. [17].

\begin{tabular}{|c|c|c|c|c|}
\hline Class & $\begin{array}{l}\text { Mean delay } \\
\text { upper bound }\end{array}$ & Delay variance & Loss ratio & Application examples \\
\hline $\begin{array}{l}\text { Class } 0 \text { Real-time, highly interactive applications sensitive to } \\
\text { jitter }\end{array}$ & $100 \mathrm{~ms}$ & $<50 \mathrm{~ms}$ & $<1,000 \mathrm{E}-3$ & Voice over IP, Video Teleconference \\
\hline Class 1 Real-time, interactive applications sensitive to jitter & $400 \mathrm{~ms}$ & $<50 \mathrm{~ms}$ & $<1,000 \mathrm{E}-3$ & Voice over IP, Video Teleconference \\
\hline Class 2 Highly interactive transaction data & $100 \mathrm{~ms}$ & Unspecified & $<1,000 \mathrm{E}-3$ & Signaling \\
\hline Class 3 interactive transaction data & $400 \mathrm{~ms}$ & Unspecified & $<1,000 \mathrm{E}-3$ & Signaling \\
\hline Class 4 Low loss only applications & $1 \mathrm{~s}$ & Unspecified & $<1,000 \mathrm{E}-3$ & $\begin{array}{l}\text { Short transactions, bulk data, video } \\
\text { streaming }\end{array}$ \\
\hline Class 5 Unspecified applications & Unspecified & Unspecified & Unspecified & Default IP networks \\
\hline
\end{tabular}

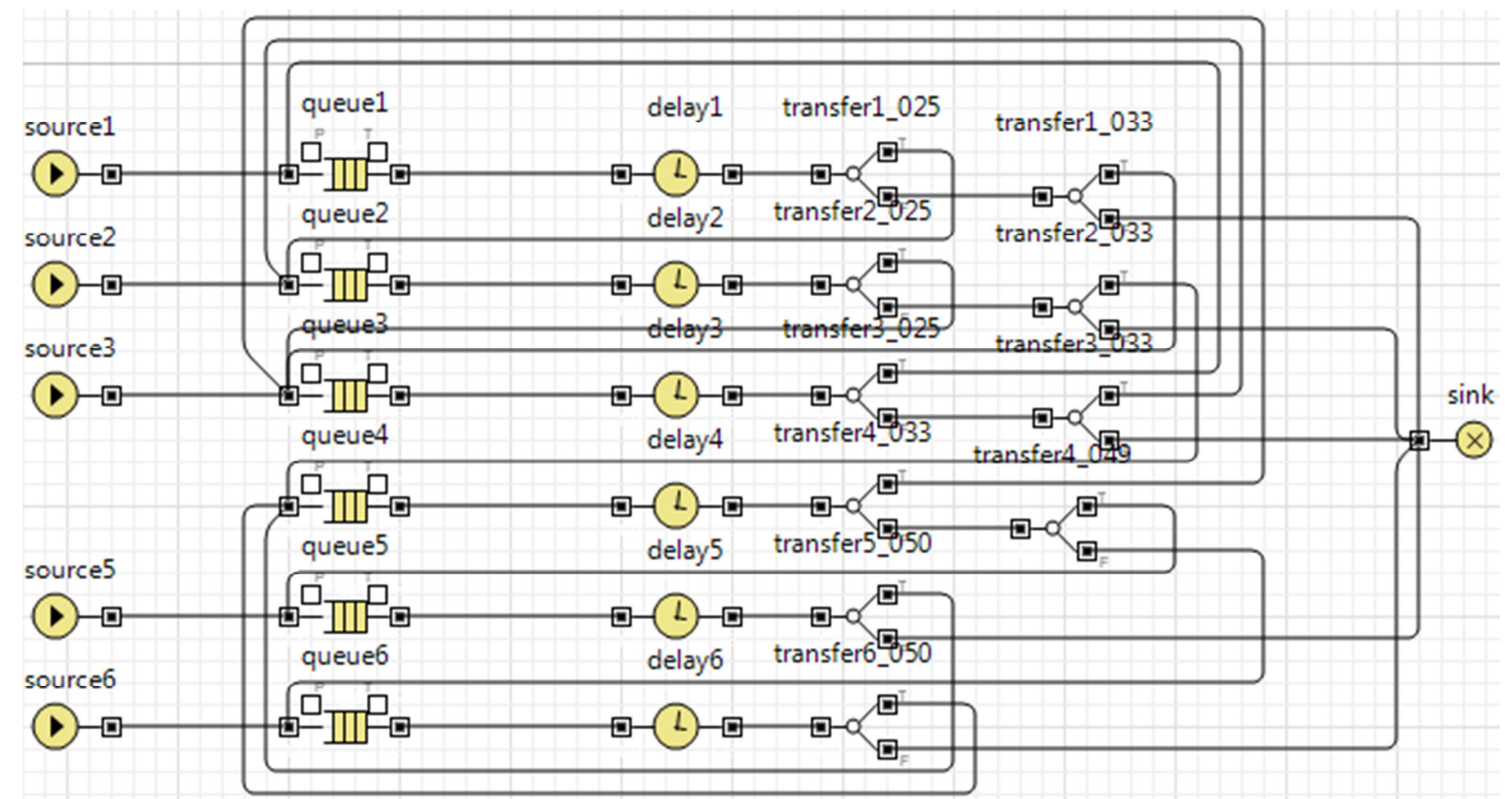

Figure 3. Telecommunication network modeled in AnyLogic. 


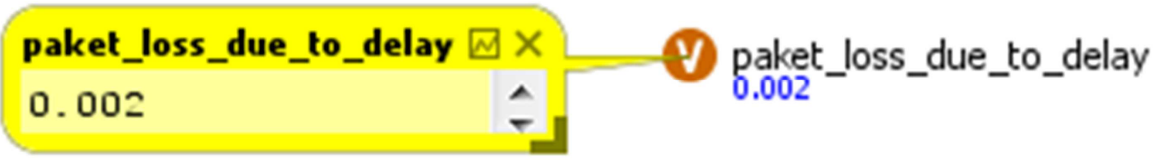

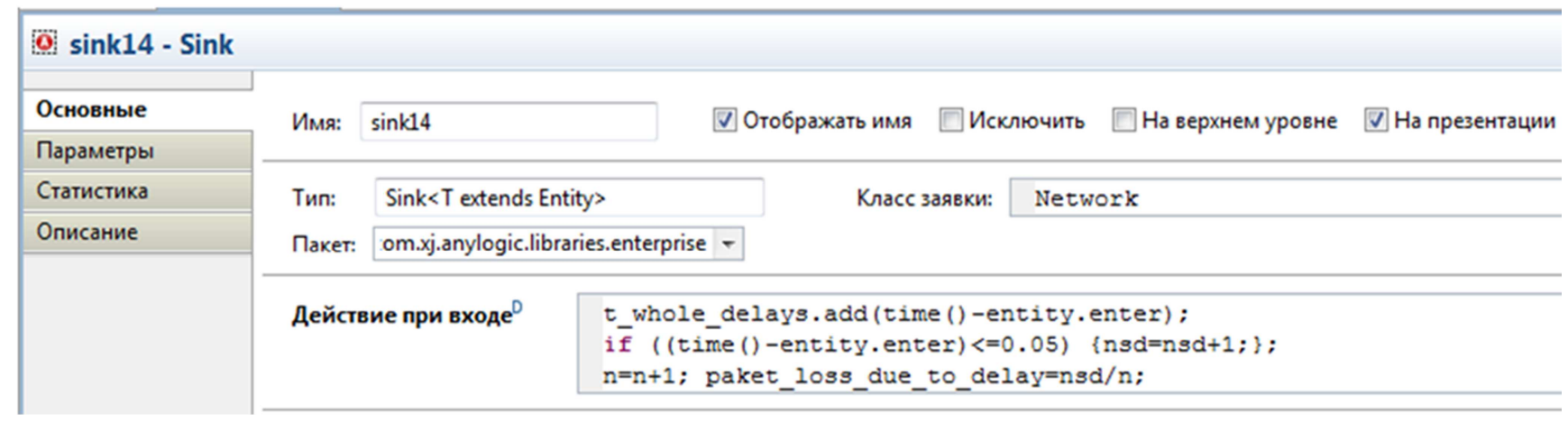

Figure 4. The sink window in AnyLogic (delay variance $\leq 50 \mathrm{~ms}$ ) and the probability of the packet loss in the network when the delay variance $\leq 50 \mathrm{~ms}$.

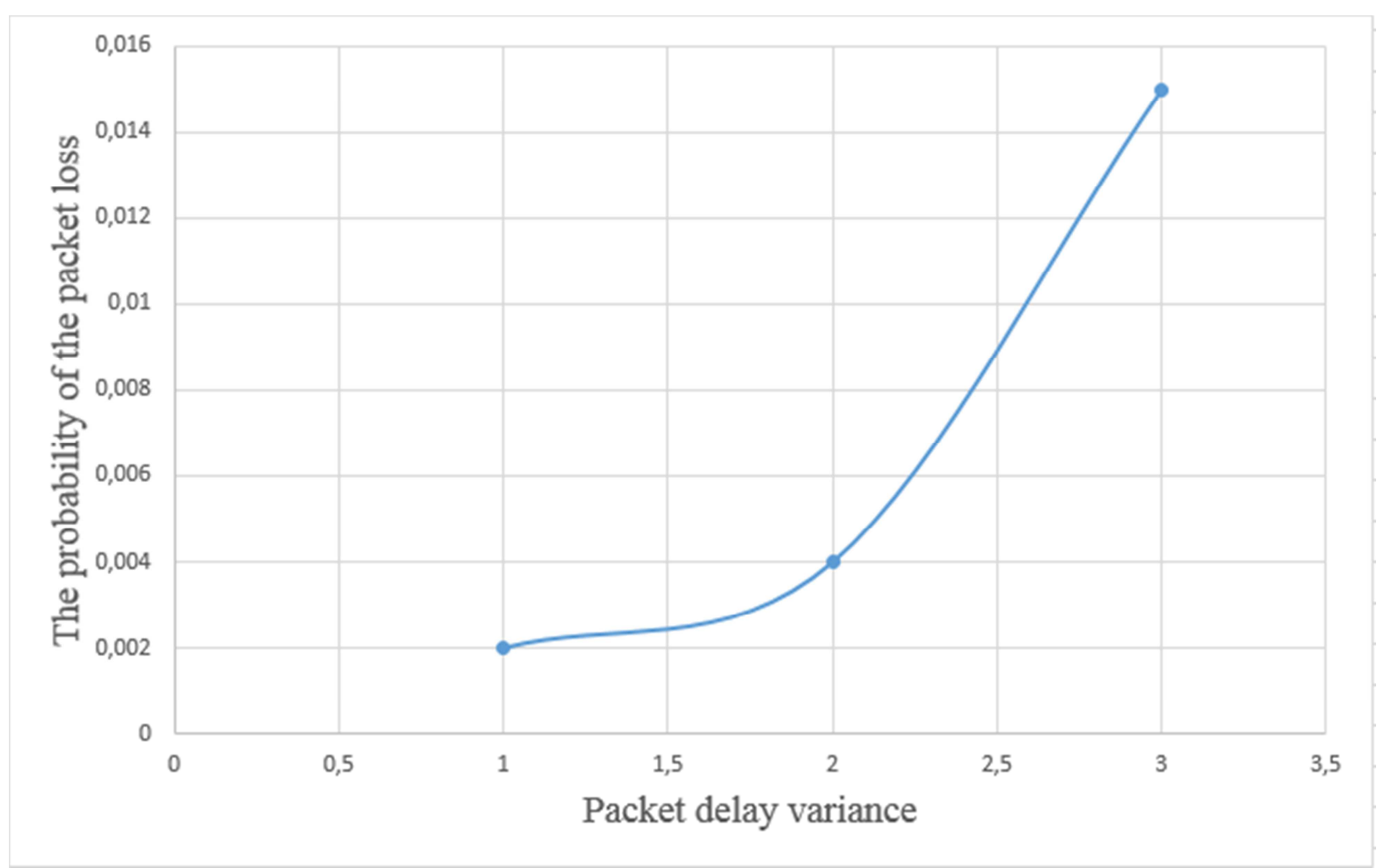

Figure 5. The relevant graph between packet delay variance and the probability of packet loss.

\section{Discussion}

The packet loss processes are simulated in two ways in this paper, the first is due to the buffer overflow and the second is due to the delay variance in infinite buffers. Furthermore the relevant line graph between packet delay variance and the probability of the packet loss is illustrated.

By modeling the processes of packet losses in telecommunication networks it can be predicted and solved the problems related to the network characteristics which may occur in the network in the near future. Also, in order to get the real results of the simulated model of the existing telecommunication network, it is demanded to just enter the original values of the network on simulated AnyLogic model.

\section{Conclusion}

In the summary of this paper may conclude as follow:

By modeling the processes of packet losses in telecommunication networks it can be predicted and solved the problems related to the network characteristics which may occur in the network in the near future. Also, in order to get the real results of the simulated model of the existing telecommunication network, it is demanded to just enter the original values of the network on simulated AnyLogic model. 


\section{References}

[1] Gunnar Karlsson. Asynchronous transfer of video. IEEE CommunicationsMagazine, 34 (8):118 \{126, August 1996.

[2] ITU, "End-user Multimedia QoS Categories", ITU-T Recommendation G. 1010, August 2001.

[3] Martin F. Arlitt and Carey L. Williamson. Web server workload characterization: The search for invariants. In Proceedings of ACM SIGMETRICS '96: Conference on Measurement and Modeling of Computer Systems, pages 126\{137, Philadelphia, PA, USA, May 1996.

[4] HariBalakrishnan, VenkatPadmanabhan, SrinivasanSeshan, and Randy H. Katz. A comparison of mechanisms for improving TCP performance over wireless links. IEEE/ACM Transactions on Networking, 5 (6):756\{769, December 1997.

[5] HariBalakrishnan, Hariharan S. Rahul, and SrinivasanSeshan. An integrated congestion management architecture for internet hosts. In Proceedings of ACM SIGCOMM '99 Conference on Applications, Technologies, Architectures, and Protocols for Computer Communication, pages $175\{187$, Cambridge, MA, USA, August1999.

[6] Paul Barford and Mark Crovella. Generating representative Web workloads fornetwork and server performance evaluation. In Proceedings of SIGMETRICS'98/PERFORMANCE '98, Joint International Conference on Measurement andModeling of Computer Systems, pages 151\{160, Madison, Wisconsin, USA, June 1998.

[7] Jan Beran. Statistics for long-memory processes, chapter 12. Monographs onstatistics and applied probability, No. 61. Chapman \& Hall, New York, NY, USA, 1994.

[8] Tim Berners-Lee, Roy T. Fielding, and HenrikFrystyk
Nielsen. Hypertext TransferProtocol $\{$ HTTP/1.0. Request for Comments 1945, Internet Engineering TaskForce, May 1996.

[9] Jean-ChrysostomeBolot. End-to-end packet delay and loss behavior in the Internet. In Proceedings of ACM SIGCOMM '93 Conference on Communications Architectures, Protocols and Applications, pages 289\{298, San Francisco, CA, USA, September 1993.

[10] Jean-ChrysostomeBolot, Sacha Fosse-Parisis, and Don Towsley. Adaptive FECbasederror control for Internet telephony. In Proceedings of IEEE INFOCOM, pages 1453 \{1460, New York, NY, USA, March 1999.

[11] Jean-ChrysostomeBolot, Thierry Turletti, and Ian Wakeman. Scalable feedbackcontrol for multicast video distribution in the Internet. In Proceedings of ACMSIGCOMM '94 Conference on Communications Architectures, Protocols and Applications, pages 58 \{67, London, UK, August 1994.

[12] Srinivasan Keshav and Rosen Sharma. Issues and trends in router design. IEEECommunications Magazine, 36 (5): $144\{151$, May 1998.

[13] Van Jacobson. Congestion avoidance and control. In SIGCOMM '88, Proceedingsof the ACM Symposium on Communications Architectures and Protocols, pages $314\{329$, Stanford, CA, USA, August 1988.

[14] Raj Jain. Congestion control in computer networks: Issues and trends. IEEENetwork, 4 (3):24 \{30, May 1990.

[15] John B. Nagle. On packet switches with in nite storage. IEEE Transactions onCommunications, COM-35 (4):435 \{438, April 1987.

[16] Vern Paxson. Measurements and analysis of end-to-end Internet dynamics. PhDthesis, University of California, Berkeley, Berkeley, CA, USA, April 1997.

[17] ITU Recommendation Y. 154. 